\title{
PERIODIC SOLUTIONS OF A GALACTIC POTENTIAL
}

\author{
JAUME LLIBRE ${ }^{1}$, DANIEL PAŞCA ${ }^{2}$ AND CLAUDIÀ VALLS ${ }^{3}$
}

\begin{abstract}
We study analytically the periodic solutions of a Hamiltonian in $\mathbb{R}^{6}$ given by the kinetic energy plus a galactic potential, using averaging theory of first order. The model perturbs a harmonic oscillator, and has been extensively used in order to describe local motion in galaxies near an equilibrium point.
\end{abstract}

\section{Introduction And StATEMEnt of the MAin RESUlts}

We consider the Hamiltonian

$$
H=H\left(x, y, z, p_{x}, p_{y}, p_{z}\right)=\frac{1}{2}\left(p_{x}^{2}+p_{y}^{2}+p_{z}^{2}\right)+V(x, y, z),
$$

where the potential $V=V(x, y, z)$ is given by

$$
V=\frac{\omega^{2}}{2}\left(x^{2}+y^{2}+z^{2}\right)-\varepsilon\left(a\left(x^{4}+y^{4}+z^{4}\right)+2 b\left(x^{2} y^{2}+x^{2} z^{2}+y^{2} z^{2}\right)\right),
$$

where $\varepsilon$ is a small parameter (the perturbation strength), and $a$ and $b$ are parameters. Here $\omega$ is the common unperturbed frequency of the oscillations along the $x, y$ and $z$ axis. The potential $V$ is a 3 dimensional perturbed harmonic oscillator and describes local motion in the central parts of a galaxy. It is called galactic potential. Such local 3-dimensional potentials appear after the expansion of global galactic potentials in a Taylor series near a stable equilibrium point and have been used by many authors in order to describe local motion in galaxies, see for instance Deprit and Elipe [3], Caranicolas [2], Elipe and Deprit [4], Elipe [5], Arribas et al. [1], Zotos [7, 8, 9], ...

It is not restrictive, rescaling the variables $(x, y, z)$ if necessary, to take $\omega=1$. In short we shall study the periodic solutions of the

2010 Mathematics Subject Classification. Primary 34C10, 34C25.

Key words and phrases. galactic potential, periodic solution, averaging theory. 
Hamiltonian system

$$
\begin{aligned}
& \dot{x}=p_{x}, \\
& \dot{p}_{x}=x+\varepsilon\left(4 a x^{3}+2 b\left(2 x y^{2}+2 x z^{2}\right)\right), \\
& \dot{y}=p_{y}, \\
& \dot{p}_{y}=-y+\varepsilon\left(4 a y^{3}+2 b\left(2 x^{2} y+2 y z^{2}\right)\right), \\
& \dot{z}=p_{z}, \\
& \dot{p}_{z}=-z+\varepsilon\left(4 a z^{3}+2 b\left(2 x^{2} z+2 y^{2} z\right)\right),
\end{aligned}
$$

with Hamiltonian

$$
\begin{aligned}
H= & \frac{1}{2}\left(p_{x}^{2}+p_{y}^{2}+p_{z}^{2}\right)+\frac{1}{2}\left(x^{2}+y^{2}+z^{2}\right) \\
& -\varepsilon\left(a\left(x^{4}+y^{4}+z^{4}\right)+2 b\left(x^{2} y^{2}+x^{2} z^{2}+y^{2} z^{2}\right)\right) .
\end{aligned}
$$

As far as we know there are no rigorous analytic studies of the existence of periodic solutions for the Hamiltonian system (1). The only studies in this direction are numerical or pseudo-numerical, (see [2] and the references therein). Here we shall study the periodic solutions of system (1) analytically by using the averaging theory, see for more details section 2. In short, the averaging method, is the procedure of replacing a vector field by its average (over time or an angular variable) with the goal to obtain asymptotic approximations to the original system and to obtain periodic solutions.

We shall provide sufficient conditions on the two parameters $a$ and $b$ of the Hamiltonian system (1) to guarantee the existence of continuous families of periodic solutions parameterized by the energy, and these families are given explicitly up to first order in the small parameter $\varepsilon$.

After equilibrium points which can be considered as being trivial, periodic solutions are the most simple non-trivial solutions of a differential system. Their study is of special interest because the motion in their neighborhood can be determined by their kind of stability. We shall use the averaging theory of first order as it is stated in section 2 for studying the periodic solutions of the Hamiltonian system (1) in every energy level $H=h>0$.

The following table which provides 15 periodic solutions of the unperturbed Hamiltonian system (1), i.e. system (1) with $\varepsilon=0$, will be used in the statement of our main result, Theorem 1.

(i) $\sqrt{2 h}(0, \cos t, 0,0,-\sin t, 0)$.

(ii) $\sqrt{2 h}(0,0, \cos t, 0,0,-\sin t)$.

(iii) $\sqrt{2 h}(0, \cos t, \cos t, 0,-\sin t,-\sin t)$. 
(iv) $\sqrt{2 h}(0, \cos t, \cos (t+\sigma), 0,-\sin t,-\sin (t+\sigma))$ where $\sigma=\arccos ((3 a-b) /(2 b))$.

(v) $\sqrt{2 h}(\cos t, 0,0,-\sin t, 0,0)$.

(vi) $\sqrt{\frac{2 h}{3}}\left(\cos t, \sin \left(\frac{\pi}{6}-t\right),-\sin \left(\frac{\pi}{6}+t\right),-\sin t,-\cos \left(\frac{\pi}{6}-t\right),-\cos \left(\frac{\pi}{6}+t\right)\right)$;

(vii) $\sqrt{\frac{2 h}{3}}\left(\cos t,-\sin \left(\frac{\pi}{6}-t\right),-\sin \left(\frac{\pi}{6}+t\right),-\sin t, \cos \left(\frac{\pi}{6}-t\right),-\cos \left(\frac{\pi}{6}+t\right)\right)$;

(viii) $\sqrt{\frac{2 h}{3}}\left(\cos t,-\sin \left(\frac{\pi}{6}-t\right), \sin \left(\frac{\pi}{6}-t\right),-\sin t,-\cos \left(\frac{\pi}{6}+t\right),-\cos \left(\frac{\pi}{6}-t\right)\right)$;

(ix) $\sqrt{\frac{2 h}{3}}\left(\cos t, \sin \left(\frac{\pi}{6}-t\right), \sin \left(\frac{\pi}{6}-t\right),-\sin t, \cos \left(\frac{\pi}{6}+t\right),-\cos \left(\frac{\pi}{6}-t\right)\right) ;$

(x) $\sqrt{\frac{2 h}{3}}\left(\cos t, \sin \left(\frac{\pi}{6}-t\right), \sin \left(\frac{\pi}{6}+t\right),-\sin t,-\cos \left(\frac{\pi}{6}-t\right), \cos \left(\frac{\pi}{6}+t\right)\right)$;

(xi) $\sqrt{\frac{2 h}{3}}\left(\cos t,-\sin \left(\frac{\pi}{6}-t\right), \sin \left(\frac{\pi}{6}+t\right),-\sin t, \cos \left(\frac{\pi}{6}-t\right), \cos \left(\frac{\pi}{6}+t\right)\right)$;

(xii) $\sqrt{\frac{2 h}{3}}\left(\cos t,-\sin \left(\frac{\pi}{6}+t\right),-\sin \left(\frac{\pi}{6}-t\right),-\sin t,-\cos \left(\frac{\pi}{6}+t\right), \cos \left(\frac{\pi}{6}-t\right)\right)$;

(xiii) $\sqrt{\frac{2 h}{3}}\left(\cos t, \sin \left(\frac{\pi}{6}+t\right),-\sin \left(\frac{\pi}{6}-t\right),-\sin t, \cos \left(\frac{\pi}{6}+t\right), \cos \left(\frac{\pi}{6}-t\right)\right) ;$

(xiv) $\sqrt{h}(\cos t, 0, \cos t,-\sin t, 0,-\sin t)$.

(xv) $\sqrt{h}(\cos t, 0,-\cos t,-\sin t, 0, \sin t)$.

TABLE 1. Fifteen periodic solutions of the unperturbed Hamiltonian system (1).

Our main result on the periodic solutions of the Hamiltonian system associated to the Hamiltonian system 1 is the following.

Theorem 1. Using averaging theory of first order for $\varepsilon \neq 0$ sufficiently small at every energy level $H=h>0$ of the Hamiltonian $H$ given in (2), we find for its associated Hamiltonian system (1) periodic solutions bifurcating from the periodic solutions of Table 1. The number of these periodic solutions depends on the parameters $(a, b)$ of the system. If the parameters $(a, b) \neq(0,0)$ belong to the straight lines $b=\mu a$

(a) with $\mu \in\{0,1\}$ we do not find periodic solutions;

(b) with $\mu \in[-3,0) \cup(0,1)$ we find 8 periodic solutions bifurcating from the periodic solutions $(v i), \ldots$, (xiii) of Table 1 ;

(c) with $\mu \in(1,3)$ we find 15 periodic solutions bifurcating from the 15 periodic solutions of Table 1 .

(d) with $\mu \in[3, \infty) \cup(-\infty,-3)$ and for the points $(0, b) \neq(0,0)$ we find 12 periodic solutions bifurcating from all the periodic solutions of Table 1 except from the periodic solutions (i), (ii) and $(v)$. 
Theorems 1 is proved in section 3 .

Using statement (b) of Theorem 2 it would be possible to compute the linear stability or instability of the periodic solutions of Theorem 1. This stability or instability depends of the eigenvalues of a $4 \times 4$ matrix, but the expressions of these eigenvalues are huge in function of the parameters $a$ and $b$, and it is not easy to control the sign of their real parts in function of the parameters $a$ and $b$. Since they are easy to compute with the help of an algebraic manipulator as mathematica or mapple we do not provide them here.

\section{THE AVERAGING THEORY OF FIRST ORDER}

Now we shall provide the basic results from averaging theory that we need for proving the results of this paper.

The following theorem provides a first order approximation for the periodic solutions of a periodic differential system, for the proof see Theorems 11.5 and 11.6 of Verhulst [6].

Consider the differential equation

$$
\dot{\mathbf{x}}=\varepsilon F_{1}(t, \mathbf{x})+\varepsilon^{2} F_{2}(t, \mathbf{x}, \varepsilon), \quad \mathbf{x}(0)=\mathbf{x}_{0}
$$

with $\mathbf{x} \in D$, where $D$ is an open subset of $\mathbb{R}^{n}, t \geq 0$. Moreover we assume that both $F_{1}(t, \mathbf{x})$ and $F_{2}(t, \mathbf{x}, \varepsilon)$ are $T$-periodic in $t$. We also consider in $D$ the averaged differential equation

$$
\dot{\mathbf{y}}=\varepsilon f_{1}(\mathbf{y}), \quad \mathbf{y}(0)=\mathbf{x}_{0},
$$

where

$$
f_{1}(\mathbf{y})=\frac{1}{T} \int_{0}^{T} F_{1}(t, \mathbf{y}) d t
$$

Under certain conditions, equilibrium solutions of the averaged equation turn out to correspond with $T$-periodic solutions of equation (3).

Theorem 2. Consider the two initial value problems (3) and (4). Suppose:

(i) $F_{1}$, its Jacobian $\partial F_{1} / \partial x$, its Hessian $\partial^{2} F_{1} / \partial x^{2}, F_{2}$ and its Jacobian $\partial F_{2} / \partial x$ are defined, continuous and bounded by a constant independent of $\varepsilon$ in $[0, \infty) \times D$ and $\varepsilon \in\left(0, \varepsilon_{0}\right]$.

(ii) $F_{1}$ and $F_{2}$ are $T$-periodic in $t$ ( $T$ independent of $\varepsilon$ ).

Then the following statements hold.

(a) If $p$ is an equilibrium point of the averaged equation (4) and

$$
\left.\operatorname{det}\left(\frac{\partial f_{1}}{\partial \mathbf{y}}\right)\right|_{\mathbf{y}=p} \neq 0,
$$


then there exists a $T$-periodic solution $\varphi(t, \varepsilon)$ of equation (3) such that $\varphi(0, \varepsilon) \rightarrow p$ as $\varepsilon \rightarrow 0$.

(b) The stability or instability of the limit cycle $\varphi(t, \varepsilon)$ is given by the stability or instability of the equilibrium point $p$ of the averaged system (4). In fact the singular point $p$ has the stability behavior of the Poincaré map associated to the limit cycle $\varphi(t, \varepsilon)$.

For more details on Theorem 2 see Section 6.3 and 11.8 in [6].

\section{Proof of Theorem 1}

Let $\mathbb{R}^{+}=[0, \infty)$ and $\mathbb{S}^{1}$ the circle. We do the change of variables $\left(x, y, z, p_{x}, p_{y}, p_{z}\right) \rightarrow(r, \theta, \rho, \alpha, R, \beta) \in \mathbb{R}^{+} \times \mathbb{S}^{1} \times \mathbb{R}^{+} \times \mathbb{S}^{1} \times \mathbb{R}^{+} \times \mathbb{S}^{1}$ defined by

$$
\begin{array}{lll}
x=r \cos \theta, & y=\rho \cos (\theta+\alpha), & z=R \cos (\theta+\beta), \\
p_{x}=r \sin \theta, & p_{y}=\rho \sin (\theta+\alpha), & p_{z}=R \sin (\theta+\beta) .
\end{array}
$$

This change of variables are well defined when $r>0, \rho>0$ and $R>0$.

The differential system in the new variables become

$$
\begin{aligned}
& \dot{r}=\varepsilon 2 r \sin (2 \theta)\left(a r^{2} \cos ^{2} \theta+b \rho^{2} \cos ^{2}(\alpha+\theta)+b R^{2} \cos ^{2}(\beta+\theta)\right) \text {, } \\
& \dot{\theta}=-1+\varepsilon 4 \cos ^{2} \theta\left(a r^{2} \cos ^{2} \theta+b \rho^{2} \cos ^{2}(\alpha+\theta)\right. \\
& \left.+b R^{2} \cos ^{2}(\beta+\theta)\right) \text {, } \\
& \dot{\rho}=\varepsilon 4 \rho \sin (\alpha+\theta) \cos (\alpha+\theta)\left(b r^{2} \cos ^{2} \theta+a \rho^{2} \cos ^{2}(\alpha+\theta)\right. \\
& \left.+b R^{2} \cos ^{2}(\beta+\theta)\right) \text {, } \\
& \dot{\alpha}=\varepsilon 4\left(a \rho^{2} \cos ^{4}(\alpha+\theta)+b R^{2} \cos ^{2}(\alpha+\theta) \cos ^{2}(\beta+\theta)\right. \\
& -a r^{2} \cos ^{4} \theta+b \cos ^{2} \theta\left(\left(r^{2}-\rho^{2}\right) \cos ^{2}(\alpha+\theta)\right. \\
& \left.\left.-R^{2} \cos ^{2}(\beta+\theta)\right)\right) \\
& \dot{R}=\varepsilon 4 R \sin (\beta+\theta) \cos (\beta+\theta)\left(b r^{2} \cos ^{2} \theta+b \rho^{2} \cos ^{2}(\alpha+\theta)\right. \\
& \left.+a R^{2} \cos ^{2}(\beta+\theta)\right) \text {, } \\
& \dot{\beta}=\varepsilon 4\left(b \rho^{2} \cos ^{2}(\alpha+\theta) \cos ^{2}(\beta+\theta)+a R^{2} \cos ^{4}(\beta+\theta)\right. \\
& -a r^{2} \cos ^{4} \theta-b \cos ^{2} \theta\left(\rho^{2} \cos ^{2}(\alpha+\theta)\right. \\
& \left.\left.+\left(R^{2}-r^{2}\right) \cos ^{2}(\beta+\theta)\right)\right),
\end{aligned}
$$


with Hamiltonian

$$
\begin{aligned}
H= & \frac{1}{2}\left(r^{2}+R^{2}+\rho^{2}\right)+\varepsilon\left(-2 b\left(r^{2} \rho^{2} \cos ^{2} \theta \cos ^{2}(\alpha+\theta)\right.\right. \\
& \left.+R^{2}\left(r^{2} \cos ^{2} \theta+\rho^{2} \cos ^{2}(\alpha+\theta)\right) \cos ^{2}(\beta+\theta)\right) \\
& \left.-a\left(r^{4} \cos ^{4} \theta+\rho^{4} \cos ^{4}(\alpha+\theta)+R^{4} \cos ^{4}(\beta+\theta)\right)\right) .
\end{aligned}
$$

In order that the right-hand side of the differential system (6) be periodic with respect to the independent variable, we change the old independent variable $t$ by the new independent variable $\theta$, for obtaining the periodicity necessary for applying the averaging theory. Dividing system (6) by $\dot{\theta}$ and omitting the $\dot{\theta}$ equation, system (6) goes over to

$$
\begin{aligned}
& r^{\prime}=-\varepsilon 2 r \sin (2 \theta)\left(a r^{2} \cos ^{2} \theta+b \rho^{2} \cos ^{2}(\alpha+\theta)\right. \\
&\left.+b R^{2} \cos ^{2}(\beta+\theta)\right)+O\left(\varepsilon^{2}\right), \\
& \rho^{\prime}=-\varepsilon 4 \rho \sin (\alpha+\theta) \cos (\alpha+\theta)\left(b r^{2} \cos ^{2} \theta+a \rho^{2} \cos ^{2}(\alpha+\theta)\right. \\
&\left.+b R^{2} \cos ^{2}(\beta+\theta)\right)+O\left(\varepsilon^{2}\right), \\
& \alpha^{\prime}= \varepsilon 4\left(b \cos ^{2} \theta\left(\left(\rho^{2}-r^{2}\right) \cos ^{2}(\alpha+\theta)+R^{2} \cos ^{2}(\beta+\theta)\right)\right. \\
& \quad-\cos ^{2}(\alpha+\theta)\left(a \rho^{2} \cos ^{2}(\alpha+\theta)+b R^{2} \cos ^{2}(\beta+\theta)\right) \\
&\left.\quad+a r^{2} \cos ^{4} \theta\right)+O\left(\varepsilon^{2}\right), \\
& R^{\prime}=-\varepsilon 4 R \sin ^{2}(\beta+\theta) \cos (\beta+\theta)\left(b r^{2} \cos ^{2} \theta+b \rho^{2} \cos ^{2}(\alpha+\theta)\right. \\
&\left.+a R^{2} \cos ^{2}(\beta+\theta)\right)+O\left(\varepsilon^{2}\right), \\
& \beta^{\prime}=\varepsilon 4\left(-\cos ^{2}(\beta+\theta)\left(b \rho^{2} \cos ^{2}(\alpha+\theta)+a R^{2} \cos ^{2}(\beta+\theta)\right)\right. \\
& \quad+b \cos ^{2} \theta\left(\rho^{2} \cos ^{2}(\alpha+\theta)+\left(R^{2}-r^{2}\right) \cos ^{2}(\beta+\theta)\right) \\
&\left.\quad+a r^{2} \cos ^{4} \theta\right)+O\left(\varepsilon^{2}\right),
\end{aligned}
$$

where the prime denotes the derivative with respect to the new independent variable $\theta$. System (7) is $2 \pi$-periodic in the variable $\theta$.

In order that the application of the Averaging Theorem provides information we must fix the value of the first integral at $H=h>0$, otherwise the periodic solutions are not isolated (because they leave on cylinders of periodic solutions parameterized by the energy $h$ ) and the Jacobian of Theorem 2 would be zero. 
Solving the equation $H=h>0$ with respect to the variable $\rho$ we obtain

$$
\begin{aligned}
\rho= & \sqrt{2 h-r^{2}-R^{2}}+\frac{\varepsilon}{\sqrt{2 h-r^{2}-R^{2}}}\left(a\left(-2 h+r^{2}+R^{2}\right)^{2} \cos ^{4}(\alpha+\theta)\right. \\
& +a r^{4} \cos ^{4} \theta-2 b R^{2}\left(-2 h+r^{2}+R^{2}\right) \cos ^{2}(\alpha+\theta) \cos ^{2}(\beta+\theta) \\
& +a R^{4} \cos ^{4}(\beta+\theta)+2 b r^{2} \cos ^{2} \theta\left(\left(2 h-r^{2}-R^{2}\right) \cos ^{2}(\alpha+\theta)\right. \\
& \left.\left.+R^{2} \cos ^{2}(\beta+\theta)\right)\right)+O\left(\varepsilon^{2}\right) .
\end{aligned}
$$

Then substituting $\rho$ into equations (7) we obtain the following system

$$
\begin{aligned}
r^{\prime}= & -\varepsilon 2 r \sin (2 \theta)\left(a r^{2} \cos ^{2} \theta+b\left(2 h-r^{2}-R^{2}\right) \cos ^{2}(\alpha+\theta)\right. \\
& \left.+b R^{2} \cos ^{2}(\beta+\theta)\right)+O\left(\varepsilon^{2}\right)=F_{1 r}+O\left(\varepsilon^{2}\right), \\
\alpha^{\prime}= & \varepsilon 4\left(a r^{2} \cos ^{4} \theta+a\left(-2 h+r^{2}+R^{2}\right) \cos ^{4}(\alpha+\theta)\right. \\
& -b R^{2} \cos ^{2}(\alpha+\theta) \cos ^{2}(\beta+\theta)+b \cos ^{2} \theta\left(\left(2 h-2 r^{2}-R^{2}\right) \cos ^{2}(\alpha+\theta)\right. \\
& \left.\left.+R^{2} \cos ^{2}(\beta+\theta)\right)\right)+O\left(\varepsilon^{2}\right)=F_{1 \alpha}+O\left(\varepsilon^{2}\right), \\
R^{\prime}= & -\varepsilon 2 R \sin (2(\beta+\theta))\left(b r^{2} \cos ^{2} \theta+b\left(2 h-r^{2}-R^{2}\right) \cos ^{2}(\alpha+\theta)\right. \\
& \left.+a R^{2} \cos ^{2}(\beta+\theta)\right)+O\left(\varepsilon^{2}\right)=F_{1 R}+O\left(\varepsilon^{2}\right), \\
\beta^{\prime}= & \varepsilon 4\left(a r^{2} \cos ^{4} \theta+\cos ^{2}(\beta+\theta)\left(b\left(-2 h+r^{2}+R^{2}\right) \cos ^{2}(\alpha+\theta)\right.\right. \\
& \left.-a R^{2} \cos ^{2}(\beta+\theta)\right)+b \cos ^{2} \theta\left(\left(2 h-r^{2}-R^{2}\right) \cos ^{2}(\alpha+\theta)\right. \\
& \left.\left.+\left(R^{2}-r^{2}\right) \cos ^{2}(\beta+\theta)\right)\right)+O\left(\varepsilon^{2}\right)=F_{1 \beta}+O\left(\varepsilon^{2}\right) .
\end{aligned}
$$

We see that this differential system has the canonical form (3) for applying the averaging theory and satisfies the assumptions of Theorem 2 for $|\varepsilon|>0$ sufficiently small, with $T=2 \pi$ and $F_{1}=\left(F_{1 r}, F_{1 \alpha}, F_{1 R}, F_{1 \beta}\right)$ which are analytical functions.

Averaging the function $F_{1}$ with respect to the variable $\theta$ we obtain after easy integrations but tedious ones the following averaged function

$$
\begin{aligned}
f_{1}(r, \alpha, R, \beta)= \\
\quad=\left(f_{1 r}(r, \alpha, R, \beta), f_{1 \alpha}(r, \alpha, R, \beta), f_{1 R}(r, \alpha, R, \beta), f_{1 \beta}(r, \alpha, R, \beta)\right) \\
\quad=\int_{0}^{2 \pi}\left(F_{1 r}, F_{1 \alpha}, F_{1 R}, F_{1 \beta}\right) d \theta,
\end{aligned}
$$


where

$$
\begin{aligned}
f_{1 r}= & \frac{1}{2} b r\left(\left(2 h-r^{2}-R^{2}\right) \sin (2 \alpha)+R^{2} \sin (2 \beta)\right), \\
f_{1 \alpha}= & \frac{1}{2}\left(\left(2 h-2 r^{2}-R^{2}\right)(-3 a+2 b+b \cos (2 \alpha))\right. \\
& \left.+2 b R^{2} \cos (2 \beta) \sin ^{2} \alpha-b R^{2} \sin (2 \alpha) \sin (2 \beta)\right), \\
f_{1 R}= & \frac{1}{2} b R\left(2 h-r^{2}-R^{2}\right) \sin (2(\alpha-\beta))-r^{2} \sin (2 \beta), \\
f_{1 \beta}= & \frac{1}{2}\left(\left(r^{2}-R^{2}\right)(3 a-2 b-b \cos (2 \beta))\right. \\
& \left.-2 b\left(2 h-r^{2}-R^{2}\right) \sin (2 \alpha-\beta) \sin \beta\right) .
\end{aligned}
$$

We have to find the zeros $\left(r^{*}, \alpha^{*}, R^{*}, \beta^{*}\right)$ of $f_{1}(r, \alpha, R, \beta)$ and to check that the Jacobian determinant at these points is not zero, i.e.,

$$
\operatorname{det}\left(\left.\frac{\partial\left(f_{1 r}, f_{1 \alpha}, f_{1 R}, f_{1 \beta}\right)}{\partial(r, \alpha, R, \beta)}\right|_{(r, \alpha, R, \beta)=\left(r^{*}, \alpha^{*}, R^{*}, \beta^{*}\right)}\right) \neq 0 .
$$

Note that if $b=0$ then $f_{1 r} \equiv 0$, and consequently the Jacobian (9) will be zero, and Theorem 2 does not provide any information on the existence of periodic solutions. Hence in what follows we assume that $b \neq 0$.

From $f_{1 r}=0$ we obtain that either $r=0$, or $r=\sqrt{K}$ if $K=$ $2 h-R^{2}+R^{2} \frac{\sin (2 \beta)}{\sin (2 \alpha)}>0$. Now we shall study the solutions of $f_{1 \alpha}=$ $f_{1 R}=f_{1 \beta}=0$ given in (8) distinguishing these two cases.

Case 1: $r=0$. In this case $f_{1 R}=\frac{1}{2} b R\left(2 h-R^{2}\right) \sin (2(\alpha-\beta))$. From $f_{1 R}=0$ we get the following subcases:

Subcase 1.1: $R=0$. Then after tedious but easy computations we obtain that the solutions of the system $f_{1 \alpha}=f_{1 \beta}=0$ in the variables $\alpha$ and $\beta$ are $\alpha= \pm \arccos ((3 a-2 b) / b) / 2$ if $(3 a-2 b) / b \in[-1,1]$, and for each of these two values of $\alpha$ we have that $\beta$ can take values in the set

$$
S=\left\{0, \pi, \pm \arccos \left(\frac{3 a-2 b}{b}\right), \pm \arccos \left(\frac{2 b-3 a}{b}\right)\right\} .
$$

For all these solutions $(\alpha, \beta)$ the determinant $(9)$ takes the value $36\left(3 a^{2}-\right.$ $\left.4 a b+b^{2}\right)^{2} h^{4}$, which is different from zero if and only if $b \notin\{0, a, 3 a\}$ because $h>0$. Now going back through the change of variables (5) and taking into account that $\dot{\theta}=-1$ when $\varepsilon=0$, it follows 
that all these solutions correspond to different initial conditions of the same periodic solution. In short, by Theorem 2 in this subcase for $\varepsilon \neq 0$ sufficiently small there is one periodic solution $\gamma_{\varepsilon}(t)=$ $\left(x(t, \varepsilon), y(t, \varepsilon), z(t, \varepsilon), p_{x}(t, \varepsilon), p_{y}(t, \varepsilon), p_{z}(t, \varepsilon)\right)$ in each Hamiltonian level $H=h>0$ if $b \notin\{0, a, 3 a\}$ and $(3 a-2 b) / b \in[-1,1]$, such that when $\varepsilon \rightarrow 0$ the solution $\gamma_{\varepsilon}(t)$ tends to the periodic solution $(i)$ of Table 1 .

We note that the set of points $(a, b)$ of the plane $\mathbb{R}^{2}$ satisfying that $b \notin\{0, a, 3 a\}$ and $(3 a-2 b) / b \in[-1,1]$ are the points $(a, b) \neq(0,0)$ which are on the straight lines $b=\mu a$ with $\mu \in(1,3)$.

Subcase 1.2: $R=\sqrt{2 h}$. Then the solutions of the system $f_{1 \alpha}=f_{1 \beta}=0$ in the variables $\alpha$ and $\beta$ are $\beta= \pm \arccos ((3 a-2 b) / b) / 2$ if $(3 a-2 b) / b \in$ $[-1,1]$, and for each of these two values of $\beta$ we have that $\alpha$ can take values of the set $S$. For all these solutions $(\alpha, \beta)$ the determinant (9) takes the value $72\left(3 a^{2}-4 a b+b^{2}\right)^{2} h^{4}$, which is different from zero if and only if $b \notin\{0, a, 3 a\}$ because $h>0$. Now going back through the change of variables, it follows that all these solutions correspond to different initial conditions of the same periodic solution. Again by Theorem 2 in this subcase for $\varepsilon \neq 0$ sufficiently small there is one periodic solution $\gamma_{\varepsilon}(t)$ in each Hamiltonian level $H=h>0$ if $b \notin$ $\{0, a, 3 a\}$ and $(3 a-2 b) / b \in[-1,1]$, such that when $\varepsilon \rightarrow 0$ the solution $\gamma_{\varepsilon}(t)$ tends to the periodic solution $(i i)$ of Table 1 .

Subcase 1.3: If $\alpha=\beta$ then solving the system $f_{1 \alpha}=0, f_{1 \beta}=0$ with respect to the variables $R$ and $\beta$ we get the two solutions $R=\sqrt{h}$ and $\beta= \pm \arccos ((3 a-b) /(2 b)) / 2$. In both cases the Jacobian determinant is equal with $9(b-a) b\left(3 a^{2}-2 a b-b^{2}\right) h^{4}$. Again going back through the change of variables, it follows that these two solutions correspond to two different periodic solutions if $(3 a-b) /(2 b) \in(-1,1)$. Therefore by Theorem 2 in this subcase for $\varepsilon \neq 0$ sufficiently small there are two periodic solutions $\gamma_{\varepsilon}^{k}(t)$ for $k=1,2$ in each Hamiltonian level $H=h>0$ if $b \notin\{0, a,-3 a\}$ and $(3 a-b) /(2 b) \in[-1,1]$, such that when $\varepsilon \rightarrow 0$ the solutions $\gamma_{\varepsilon}^{1}(t)$ and $\gamma_{\varepsilon}^{2}(t)$ tend to the periodic solutions (iii) and (iv) of Table 1 , respectively.

We note that the set of points $(a, b)$ of the plane $\mathbb{R}^{2}$ satisfying that $b \notin\{0, a,-3 a\}$ and $(3 a-b) /(2 b) \in[-1,1]$ are the points $(a, b) \neq(0,0)$ which are on the straight lines $b=\mu a$ with $\mu \in(1, \infty) \cup(-\infty,-3)$, and the points $(0, b)$ for all $b \in \mathbb{R} \backslash\{0\}$.

Subcase 1.4: $\alpha=\beta+\frac{\pi}{2}$. In these subcase we can compute the solutions of the system $f_{1 \alpha}=0, f_{1 \beta}=0$ with respect to the variables $R$ and $\beta$, 
but when we compute the determinant (9) for these solutions we always obtain that it is zero, which means that in this subcase the averaging theory given by Theorem 2 does not provide information about the periodic solutions of the differential system.

Case 2: $r=\sqrt{K}$. Then we have

$$
f_{1 R}=\frac{1}{2} b R\left(\left(-2 h+R^{2}\right) \cos \alpha-R^{2} \cos (\alpha-2 \beta)\right) \sec \alpha \sin (2 \beta) .
$$

From $f_{1 R}=0$ we get the following subcases.

Subcase 2.1: $R=0$. Then the solutions of the system $f_{1 \alpha}=f_{1 \beta}=0$ in the variables $\alpha$ and $\beta$ are $\alpha=\beta= \pm \arccos ((3 a-2 b) / b) / 2$ if $(3 a-2 b) / b \in[-1,1]$. For all these four solutions $(\alpha, \beta)$ the determinant (9) takes the value $72\left(3 a^{2}-4 a b+b^{2}\right)^{2} h^{4}$, which is different from zero if and only if $b \notin\{0, a, 3 a\}$ because $h \neq 0$. Now going back through the change of variables, it follows that all these solutions correspond to different initial conditions of the same periodic solution. Hence by Theorem 2 in this subcase for $\varepsilon \neq 0$ sufficiently small there is one periodic solution $\gamma_{\varepsilon}(t)$ in each Hamiltonian level $H=h>0$ if $b \notin\{0, a, 3 a\}$ and $(3 a-2 b) / b \in[-1,1]$, such that when $\varepsilon \rightarrow 0$ the solution $\gamma_{\varepsilon}(t)$ tends to the periodic solution (v) of Table 1.

Subcase 2.2: $R=\sqrt{2 h \cos \alpha} / \sqrt{\cos \alpha-\cos (\alpha-2 \beta)}$ if this expression is real. Now solving the system $f_{1 \alpha}=0, f_{1 \beta}=0$ we get eight solutions for $(\alpha, \beta)$, namely

$$
\begin{array}{llll}
\left(-\frac{\pi}{3},-\frac{2 \pi}{3}\right), & \left(\frac{2 \pi}{3},-\frac{2 \pi}{3}\right), & \left(-\frac{2 \pi}{3},-\frac{\pi}{3}\right), & \left(\frac{\pi}{3},-\frac{\pi}{3}\right), \\
\left(-\frac{\pi}{3}, \frac{\pi}{3}\right), & \left(\frac{2 \pi}{3}, \frac{\pi}{3}\right), & \left(-\frac{2 \pi}{3}, \frac{2 \pi}{3}\right), & \left(\frac{\pi}{3}, \frac{2 \pi}{3}\right) .
\end{array}
$$

For all these solutions we get $r=\rho=R=\sqrt{\frac{2 h}{3}}$, and the Jacobian determinants are all equal to $4(a-b)^{2} b^{2} h^{4}$, which is different from zero if and only if $b \notin\{0, a\}$ because $h>0$. Now going back through the change of variables, it follows that all these eight solutions provide different periodic solutions. So by Theorem 2 in this subcase for $\varepsilon \neq 0$ sufficiently small there are eight periodic solution $\gamma_{\varepsilon}^{k}(t)$ for $k=1, \ldots, 8$ in each Hamiltonian level $H=h>0$ if $b \notin\{0, a\}$, such that when $\varepsilon \rightarrow 0$ the solutions $\gamma_{\varepsilon}^{k}(t)$ for $k=1, \ldots, 8$ tend to the eight periodic solutions $(v i), \ldots$, (xiii) of Table 1 .

Subcase 2.3: $\sin (2 \beta)=0$. We separate this subcase in three subsubcases. 
Subsubcase 2.3.1: $\beta=0$. Then the solutions of the system $f_{1 \alpha}=$ $f_{1 \beta}=0$ in the variables $\alpha$ and $R$ are $\alpha= \pm \arccos (\sqrt{ \pm(3 a+b) / b} / 2)$ if $\sqrt{ \pm(3 a+b) / b} / 2 \in[0,1]$, and $R=\sqrt{h}$. Clearly, the square root in $\alpha$ is real only for two of these four values of $(\alpha, R)$ if $b \neq-3 a$. The determinant (9) takes in these two real solutions the value $-18(a-b)^{2} b(3 a+$ $b) h^{4}$, which is different from zero if and only if $b \notin\{0, a,-3 a\}$ because $h>0$. Now going back through the change of variables, it follows that all these two solutions correspond to different initial conditions of the same periodic solution. Hence by Theorem 2 in this subcase for $\varepsilon \neq 0$ sufficiently small there is one periodic solution $\gamma_{\varepsilon}(t)$ in each Hamiltonian level $H=h>0$ if $b \notin\{0, a,-3 a\}$ and $\sqrt{ \pm(3 a+b) /(4 b)} \in[0,1]$, such that when $\varepsilon \rightarrow 0$ the solution $\gamma_{\varepsilon}(t)$ tends to the periodic solution (xiv) of Table 1.

We note that the set of points $(a, b)$ of the plane $\mathbb{R}^{2}$ satisfying that $b \notin$ $\{0, a,-3 a\}$ and $(3 a+b) /(4 b) \in[0,1]$ are again the points $(a, b) \neq(0,0)$ which are on the straight lines $b=\mu a$ with $\mu \in(1, \infty) \cup(-\infty,-3)$, and the points $(0, b)$ for all $b \in \mathbb{R} \backslash\{0\}$.

Subsubcase 2.3.2: $\beta=\pi$. The computations are exactly as the ones of the subsubcase 2.3.1. The unique difference is that in this subcase for $\varepsilon \neq 0$ sufficiently small there is one periodic solution $\gamma_{\varepsilon}(t)$ in each Hamiltonian level $H=h>0$ if $b \notin\{0, a,-3 a\}$ and $\sqrt{ \pm(3 a+b) /(4 b)} \in$ $[0,1]$, such that when $\varepsilon \rightarrow 0$ the solution $\gamma_{\varepsilon}(t)$ tends to the periodic solution $(x v)$ of Table 1.

Subsubcase 2.3.3: $\beta= \pm \pi / 2$. In this subsubcase we can compute the solutions $(\alpha, R)$ of the system $f_{1 \alpha}=0, f_{1 \beta}=0$, but when we compute the determinant (9) for these solutions we always obtain that it is zero, so in this subsubcase Theorem 2 does not provide information about the periodic solutions of the differential system.

Finally putting together the results of theses cases and subcases it follows the statement of Theorem 1 .

\section{ACKNOWLEDGMENTS}

The first author is partially supported by a MICINN/FEDER grant number MTM2009-03437, by an AGAUR grant number 2009SGR410, by an ICREA Academia, and two FP7+PEOPLE+2012+IRSES numbers 316338 and 318999. The third author was supported by Portuguese National Funds through FCT+Fundação para a Ciência e a Tecnologia within the project PTDC/MAT/117106/2010 and by CAMGSD. 


\section{REFERENCES}

[1] M. Arribas, A. Elipe, L. Floria And A. Riaguas, Oscillators in resonance p:q:r, Chaos, Solitons \& Fractals, 27 (2006), 1220-1228.

[2] N.D. CARANICOLAS, 1:1:1 resonant periodic orbits in 3 +dimensional galactictype potentials, Astronomy \& Astrophysics 282 (1994), 34-36.

[3] A. Deprit and A. Elipe, The Lissajous transformations II - Normalization, Celestial Mechanics and Dynamical Astronomy 51 (1991), 227-250.

[4] A. Deprit And A. Elipe, Oscillators in resonance, Mechanics Research Communications 26 (1999), 635-640.

[5] A. EliPe, Complete reduction of oscillators in resonance p:q, Physical Review E 61 (2000), 6477-6484.

[6] F. Verhulst, Nonlinear Differential Equations and Dynamical Systems, Universitext Springer Verlag, 1996.

[7] E.E. Zotos, A new dynamical model for the study of galactic structure, New Astronomy 16 (2011), 391-401.

[8] E.E. ZотоS, Disks controlling chaos in a $3 D$ dynamical model for elliptical galaxies, Baltic Astronomy 20 (2011), 77-90.

[9] E.E. Zotos, A new dynamical indicator for chaos detection in galactic Hamiltonian systems, Mathematics in Engineering, Science and Aerospace Mesa 3 (2012), 163-178.

${ }^{1}$ Departament de Matemàtiques, Universitat Autònoma de Barcelona, 08193 Bellaterra, Barcelona, Catalonia, Spain

E-mail address: jllibre@mat.uab.cat

2 Department of Mathematics and Informatics, University of Oradea, University Street 1, 410087 Oradea, Romania

E-mail address: dpasca@uoradea.ro

3 Departamento de Matemática, Instituto Superior Técnico, Av. Rovisco Pais 1049-001, Lisboa, Portugal

E-mail address: cvalls@math.ist.utl.pt 